\title{
Eugène Müntz : un interlocuteur français d'Aby Warburg
}

Michela Passini

\section{(2) OpenEdition}

12 Journals

Édition électronique

URL : http://journals.openedition.org/imagesrevues/2908

DOI : 10.4000/imagesrevues.2908

ISSN : $1778-3801$

Éditeur :

Centre d'Histoire et Théorie des Arts, Groupe d'Anthropologie Historique de l'Occident Médiéval, Laboratoire d'Anthropologie Sociale, UMR 8210 Anthropologie et Histoire des Mondes Antiques

\section{Référence électronique}

Michela Passini, «Eugène Müntz : un interlocuteur français d'Aby Warburg », Images Re-vues [En ligne], Hors-série 4 | 2013, mis en ligne le 30 janvier 2013, consulté le 31 janvier 2021. URL : http:// journals.openedition.org/imagesrevues/2908; DOI : https://doi.org/10.4000/imagesrevues.2908

Ce document a été généré automatiquement le 31 janvier 2021.

Images Re-vues est mise à disposition selon les termes de la Licence Creative Commons Attribution Pas d'Utilisation Commerciale 4.0 International. 


\title{
Eugène Müntz : un interlocuteur français d'Aby Warburg
}

\author{
Michela Passini
}

Le 9 août 1903, Aby Warburg adresse à l'historien de l'art Adolph Goldschmidt une longue lettre, dans laquelle il retrace les développements de l'écriture d'une histoire de l'art depuis le XVe siècle, en se concentrant notamment sur ses évolutions les plus récentes, au tournant du XXe siècle ${ }^{1}$. Cette lettre, publiée par Ernst Gombrich dans sa biographie intellectuelle de Warburg, constitue un document essentiel dans la mesure où elle nous permet d'évaluer le positionnement de Warburg au sein d'une histoire de l'art en train de s'élaborer. Dans cet aperçu d'une "histoire de l'histoire de l'art», Warburg distingue deux grandes tendances actuelles de la discipline: les historiens de l'art "enthousiastes », qui " célèbrent la différence », les spécificités individuelles, et les historiens de l'art qui « ont pour but d'interroger les

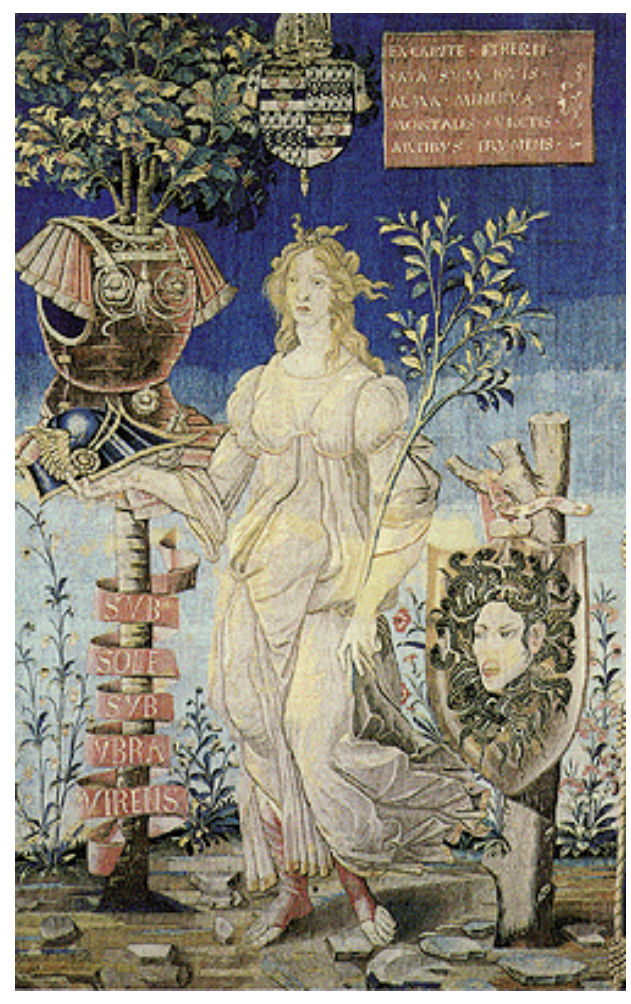
conditionnements sociologiques, c'est-àdire les obstacles universels auxquels l'individualité héroïque est confrontée ». Précisons que Warburg ne semble pas considérer ces deux tendances come opposées, mais qu'il les voit plutôt comme la continuation et le développement l'une de l'autre, même s'il prend nettement ses distances par rapport au groupe des connaisseurs à la Morelli, qu'il qualifie de « fureteurs ». 
2 Dans cette liste, comprenant presque exclusivement des représentants de la Kunstwissenschaft germanique, le seul savant français mentionné - mis à part Taine qui, mort en 1893 faisait désormais figure classique de la critique d'art - est Eugène Müntz $(1845-1902)^{2}$.

3 Figure fondamentale de médiateur entre l'histoire de l'art de langue allemande et la France, Müntz a été pour Warburg un interlocuteur privilégié. D'un point de vue purement quantitatif, Müntz est l'un des auteurs contemporains que Warburg cite le plus fréquemment dans ses écrits. Sur le plan qualitatif, Müntz représente un " pont » vers l'historiographie française, mais aussi - et c'est un point essentiel - une référence centrale et un modèle, à un moment crucial du parcours de Warburg où, autour de 1900, le savant hambourgeois posait les bases de sa méthode par ses premiers travaux sur les arts à Florence. Dès lors, il s'agira de comprendre comment l'échange entre les deux auteurs s'est construit et de dégager les éléments qui, chez Müntz, attirèrent Warburg, dans le cadre plus global d'une circulation transnationale de savoirs, de pratiques, de modèles d'historiographie, dont dépend, en ces années là, l'élaboration d'une histoire de l'art professionnelle en Europe. L'étude des relations que Warburg entretient avec Müntz permettra également de poser la question de la réception de l'œuvre warburgienne en France ou, plus précisément, d'interroger les conditions de possibilité concrètes, historiques, d'une telle réception. Ainsi, une telle étude invite à poser le problème, central dans l'étude du discours sur l'art de cette période, des traditions nationales en historiographie: de leur relative perméabilité et des représentations qui leur sont attachées.

4 Les archives de Müntz et les archives Warburg conservent en tout une dizaine de lettres échangées par les deux auteurs³. Nous sommes à la fin de 1897: Warburg travaille sur les tapisseries Valois des Uffizi, il décrit à son interlocuteur ses recherches sur les «fêtes de Bayonne » et sur les Intermezzi de Bernardo Buontalenti. On sait aujourd'hui quel rôle ces travaux sur les fêtes ont joué dans l'évolution de l'esthétique warburgienne et comment l'étude des tapisseries, comprises comme medium permettant une circulation large de motifs iconographiques, a contribué à orienter les recherches de Warburg vers une prise en compte globale des échanges artistiques entre le nord et le sud de l'Europe.

5 Mais pourquoi s'adresse-t-il à Müntz? En quoi l'œuvre du savant français représente une référence incontournable pour le plus jeune historien hambourgeois? Un premier élément de réponse nous est fourni par un texte intitulé Vom Arsenal zum Laboratorium lu le 29 décembre 1927 devant le conseil directif de la Kulturwissenschaftliche Bibliothek Warburg, et qui constitue une sorte d'autobiographie intellectuelle ${ }^{4}$. Dans ce texte, Warburg décrit un tournant essentiel dans son parcours: à la fin des années 1890, grâce à l'inventaire des collections des Médicis, publié par Müntz en 1895, Warburg put établir que les panni («peintures sur toile») de provenance flamande, présentes dans les collections des Médicis, étaient en réalité les cartons des tapisseries (perdues) que la famille Médicis avait fait tisser à Lille et qui représentaient des sujets classiques. Warburg devait rendre compte de ces découvertes dans la communication qu'il présenta au septième congrès international d'histoire de l'art de Innsbruck en septembre 1902, significativement intitulée Wappen, Stammbäume und Inventare als Hilfsmittel der Kunstgeschichte ${ }^{5}$. L'analyse des inventaires des Médicis devait également être au cœur de l'Habilitationschrift de Warburg - habilitation qu'il ne soutiendra jamais. 
Dès ses premiers ouvrages, Müntz s'était attelé à un immense travail de collecte et de publication de sources relatives à la Renaissance italienne ${ }^{6}$. Sous cet angle, son œuvre fut une référence obligée non seulement pour Warburg, mais pour tout chercheur travaillant sur la Renaissance- même pour les italiens, comme le reconnût par exemple Adolfo Venturi ${ }^{7}$. Mais Müntz ne fut pas seulement un compilateur : la recherche et la publication des sources représentèrent pour lui le premier jalon d'une recherche qui ne se limitait pas à l'évolution 'interne' des formes, mais qui se donnait pour objectif de replacer cette évolution au sein d'une histoire - beaucoup plus large - des idées esthétiques, du goût, de la culture. Les inventaires des collections, les comptes des artistes et les nombreux autres documents que Müntz étudiait, lui permettaient de comprendre et de restituer la manière dont les œuvres étaient vécues, appréciées, consommées.

7 Attiré par les thèmes de la collection, du marché, de la réception des œuvres, Müntz pratiquait une histoire de l'art où les commanditaires étaient centraux au même titre que les artistes; où une attention particulière était réservée aux mouvements intellectuels, scientifiques ou littéraires, et à leurs impact sur les arts, aux métamorphoses multiples des motives iconographiques, à la sensibilité religieuse, aux formes de la vie en société. Comme le dit Warburg dans sa lettre à Adolph Goldschmidt, Müntz interrogeait les conditionnements sociologiques dus aux coutumes et aux mœurs, auxquels l'activité artistique est soumise. Ainsi, les ouvrages de Müntz reconstituaient en détail la situation économique et politique des différents centres de la Renaissance en Italie, donnaient de vastes aperçus sur les humanités et le niveau des connaissances techniques, étudiaient le sentiment religieux, mais aussi la mode, la condition de la femme, les fêtes, la sociabilité. Müntz concevait son œuvre comme une histoire de civilisations, comme il l'écrit clairement à propos de son histoire de l'art pendant la Renaissance :

«Malgré son titre, l'ouvrage [l'Histoire de l'art pendant la Renaissance] forme en réalité une histoire de la civilisation italienne, et non pas seulement une histoire de l'art. Toute la première partie de ce volume est consacrée à des vues d'ensemble sur le sentiment national, le sentiment religieux, les mœurs et l'état d'âme dans l'Italie du XVIe siècle $»^{8}$.

Le modèle burckhardtien joue de toute évidence un rôle essentiel chez Müntz. L'Histoire de l'art pendant la Renaissance est en effet, parmi les ouvrages de Müntz, celui où l'empreinte burckhardtienne était le plus explicite. Dans l'organisation des matériaux ainsi que dans la structure même de certains chapitres Müntz s'était évidemment inspiré de Die Kultur der Renaissance in Italien. Les échanges entre les deux savants allaient d'ailleurs dans les deux sens. Ainsi, les études de Müntz sur les collections des Médicis, son édition de l'inventaire de ces collections, ont joué un rôle essentiel dans la rédaction de l'essai Burckhardt sur les collectionneurs de la Renaissance9.

9 Cette proximité de la tradition germanique de la Kulturgeschichte fit la spécificité de l'œuvre de Müntz un au sein de l'historiographie française à un moment où, en France, l'appréciation du modèle burckhardtien par les historiens de l'art était plutôt mélangée. Que l'on pense à Louis Courajod qui dans le texte des Leçons qu'il professait à l'École du Louvre, prenait explicitement ses distances par rapport à l'historien bâlois, l'enjeu étant pour Courajod d'affirmer l'existence d'une "Renaissance nationale ", nordique, antérieure à l'éclosion de la Renaissance italienne ${ }^{10}$. 
10 Müntz subit d'autant plus l'ascendant de Burckhardt qu'il était très proche de Taine, dont les ouvrages ont représenté l'un des principaux intermédiaires pour la diffusion des idées du savant bâlois en France. Bibliothécaire et responsable des collections dans cette même École des Beaux-Arts où le philosophe professait son cours d'esthétique, Müntz sera son suppléant à partir de 1885 .

11 Mais des notes de lecture, datant de ses années de formation, témoignent d'un intérêt bien plus précoce pour l'œuvre du philosophe, et font état d'une adhésion à sa méthode qu'il n'est pas excessif de définir enthousiaste :

12 «La critique de Taine ressemble à un engrenage : si vous placez un objet entre les dents des deux premières roues, elles le saisiront, le broieront et ne le lâcheront qu'après l'avoir fait passer par tous les dédales de la machine. De même, une fois que vous avez lu une page de Taine, vous êtes entrainés, vous perdez l'usage de votre raison, de votre indépendance; votre volonté est impuissante à protester; vous suivez votre guide comme si une attraction vous précipitait sur ses pas, et sa fascination survit à la lecture. Aujourd'hui encore je ne peux pas m'arracher aux idées qu'il m'a inculquées, tant elles sont profondément gravées dans ma mémoire. Il est trop puissant, il absorbe le lecteur et ne lui permet plus de le contrôler [sic], tant le charme qu'il exerce est irrésistible $»^{11}$.

13 Même si, plus tard, Müntz devait exprimer des positions plus nuancées à l'égard de la méthode tainienne, son œuvre appartient au courant d'esthétique sociologique dont Taine a été l'un des initiateurs.

14 Taine et Burckhardt ouvraient des perspectives nouvelles à la recherche en posant les assises d'un travail historique capable d'intégrer l'étude des arts dans la reconstitution des civilisations. Assumée et revendiquée par Müntz, cette filiation intellectuelle a été perçue clairement par ses contemporains : ainsi, c'est à Burckhardt que se réfère l'historien et orientaliste Henri Cordier quand il définit Müntz comme appartenant «beaucoup plus à l'école allemande d'histoire de l'art qu'à l'école française " ${ }^{12}$.

Cette familiarité de Müntz avec la Kulturgeschichte germanique, en plus des sujets qu'il a traités, peut expliquer le fait que Warburg se soit senti proche de lui plus que d'autres historiens de l'art français contemporains. Si la pratique de l'histoire de l'art comme histoire de civilisations a représenté une première raison de l'intérêt de Warburg pour l'œuvre de Müntz, une deuxième raison doit être recherchée dans le rôle joué par Müntz dans les débats sur l'iconographie et l'iconologie autour de 1900 - rôle central, comme le montrent les documents réunis par Dieter Wuttke ${ }^{13}$, et aussi, de manière encore plus explicite, la correspondance entre Müntz et August Schmarsow, fondateur $\mathrm{du}$ Kunsthistorisches Institut de Florence et figure centrale dans la formation de Warburg ${ }^{14}$.

Déjà en 1882, Müntz avait publié des Etudes sur l'histoire de la peinture et de l'iconographie

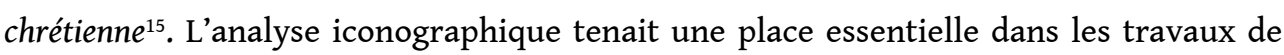
Müntz sur la Renaissance, comme outil permettant de replacer l'œuvre d'art dans un contexte culturel large. Ainsi, quand Warburg prépare le texte de sa dissertation sur la Naissance de Vénus et le Printemps de Botticelli (1892), c'est à Müntz qu'il se réfère à propos de la Pallas Athéna représentée dans cette tapisserie, que Müntz avait publiée et étudiée en 1889 dans le premier volume de son Histoire de l'art pendant la Renaissance, et qui est pour Warburg un chaînon essentiel dans sa reconstitution des relations unissant Botticelli au Politien ${ }^{16}$. 
17 Müntz sera d'ailleurs amené par ses recherches à se concentrer sur des thèmes iconographiques d'origine antique, qui avaient survécu dans le folklore et dans l'art populaire. Ainsi, entre la fin des années 1880 et le début des années 1890, il publia une série d'articles sur « Les Légendes du Moyen-Âge dans l'art de la Renaissance » dans la Revue des traditions populaires (organe de la Société des traditions populaires du Musée d'ethnographie du Trocadéro, dont Müntz était membre) fondée par l'ethnologue et folkloriste Paul Sébillot en 1882. Un de ces articles était consacré à «La Légende de Trajan ", que Warburg étudiera également ${ }^{17}$.

18 Ces travaux faisaient de Müntz une figure centrale dans les nouvelles études iconographiques. Les chercheurs plus jeunes qui, vers le milieu des années 1880, entreprenaient des travaux de ce type s'adressent à lui : sa correspondance comprend des lettres de Henry Thode, auteur d'un volume sur François d'Assise et les origines de la Renaissance en Italie, qui présente, entre autres, une étude de l'iconographie du saint $^{18}$; de Josef Strzygowski, qui avait publié ses recherches sur l'iconographie du baptême de Christ $^{19}$; plus tard d'Émile Mâle ${ }^{20}$; et surtout d'August Schmarsow, qui sera, avec Müntz, l'un des fondateurs de la Société internationale des études iconographiques ${ }^{21}$.

19 La fondation de cette société est liée à un débat central dans l'élaboration de la discipline Histoire de l'art à la fin du XIX ${ }^{e}$ siècle - un débat sur l'iconographie dans le cadre d'une discussion plus large sur les outils et les pratiques de l'histoire de l'art, qui voit la communauté des chercheurs de l'époque traversée par plusieurs lignes de clivage, dont notamment celle entre les connaisseurs, attirés par les doctrines de Morelli, et des savants pratiquant une histoire de l'art plus proche de l'histoire de la culture $^{22}$. La fondation de la Société internationale des études iconographiques est également liée à l'essor des congrès internationaux d'histoire de l'art - épisode-clé de l'institutionnalisation de la discipline. C'est dans le cadre du cinquième congrès international d'histoire de l'art qui se tint à Amsterdam en septembre 1898, que Müntz présenta une communication intitulée "La Nécessité des études iconographiques", publiée en partie par Dieter Wuttke ${ }^{23}$. Dans son intervention Müntz insistait sur l'importance de reprendre ces études dans un but polémique : «Dans les sculptures et les peintures, on ne voit plus que la question de l'attribution, sans se préoccuper du sens des œuvres, voire de leur mérite $»^{24}$.

La recherche en iconographie, avance Müntz, «offrirait autant d'intérêt que les irritantes recherches d'attribution de peintures de huitième ou dixième ordre, qui passionnent trop exclusivement la critique d'art contemporaine ». Il s'agissait ici, de toute évidence, de prendre position contre la diffusion des doctrines de Giovanni Morelli (disparu en 1891) parmi de jeunes générations de chercheurs. Dans ses écrits, publiés à partir de 1874, Morelli mettait en avant une analyse stylistique indifférente tant aux sujets représentés qu'à la dimension plus largement culturelle des œuvres d'art, une méthode qui, à partir de la comparaison de détails apparemment secondaires et moins connotées par une intentionnalité stylistique - les fameuses planches comparant les oreilles ou les ongles -, déboucherait sur des attributions fiables. Autour du milieu des années 1880, Müntz avait été l'un des adversaires les plus acharnés de Morelli. Dans sa campagne contre le connaisseur italien, il était soutenu par Schmarsow, qui, dans des lettres passionnées, commentait avec malveillance les "neuesten Expectorationen der Morellisten " et peignait dans des tons sombres la diffusion de la «Lermolieffsche Krankheit» (la maladie lermolieffienne, Lermolieff 
étant le pseudonyme sous lequel Morelli publiait ses écrits) ${ }^{25}$. La fondation de la Société internationale des études iconographiques se situe donc, entre autres, dans le contexte d'une réaction à l'« attributionnisme » morellien.

21 La correspondance Schmarsow-Müntz pour les années 1898-1900 nous renseigne sur les développements pratiques de la constitution de la Société. Müntz fut proposé par Schmarsow comme président; Schmarsow lui-même revêtait la fonction de viceprésident ; Conrad de Mandach était le secrétaire. Dans la correspondance SchmarsowMüntz apparaissent aussi plusieurs noms d'historiens de l'art de différents pays qui devaient être associés: Johan Jacob Tikkanen, Adolfo Venturi, Julius von Schlosser, Henry Thode entre autres.

En avril 1901, Müntz présentait la Société internationale des études iconographiques, à Paris, devant l'Académie des Inscriptions et Belles-Lettres. L'année suivante, à l'occasion du septième Congrès international d'histoire de l'art - le même où Warburg lut son interventions sur les inventaires Médicis -, Conrad de Mandach présentait les statuts et le programme de la Société, programme dont Müntz était l'auteur ${ }^{26}$.

C'est un texte court, mais capital: Müntz prônait une histoire de l'art comme composante essentielle de l'histoire de la culture et insistait sur la nécessité, pour l'histoire de l'art, de procéder « à l'analyse de grands courants d'idées et de sentiments auxquels, de toute nécessité, il faut qu'une œuvre d'art se rattache si elle prétend être vivante et éloquente ». Les études iconographiques étaient donc un outil pour replacer les œuvres dans leur terreau intellectuel : ainsi elles devaient prendre en compte au même titre les beaux-arts et les arts populaires, le domaine du folklore.

C'est dans ce texte que Müntz introduisait le terme « iconologie " pour caractériser ces études. Le mot « iconographie » pouvant prêter à confusion (car il s'applique aussi à l'étude des portraits), il suggérait d'utiliser le terme, plus précis, d'iconologie. On voit bien que Müntz n'attache pas de valeur particulière à cette expression, "iconologie » étant simplement, pour lui, un synonyme plus efficace d'iconographie. Cette précision de Müntz sera toutefois essentielle pour Warburg qui introduit l'adjectif iconologique dans ses écrits à partir de l'année suivante, comme l'a montré Roland Recht ${ }^{27}$.

Müntz, qui meurt en 1902, ne pourra pas développer ses recherches au sein de Société internationale des études iconographiques. En plus du rôle qu'il a joué dans la fondation des études iconographiques comme pivot d'une histoire de la culture, un dernier élément doit être pris en compte, qui semble avoir constitué un lien essentiel entre Warburg et Müntz. Müntz était particulièrement attentif aux échanges, à la transmission de formes et de modèles entre le Nord et le Sud de l'Europe : dans ses ouvrages majeurs, il fit de la France un espace de circulation et d'expérimentation pour les innovations flamandes et italiennes. Ce faisant, Müntz prenait résolument position contre une partie de l'histoire de l'art française contemporaine.

26 En France, les trois dernières décennies du XIX siècle coïncidèrent avec une phase de nationalisation accrue du discours sur l'art: l'enseignement de Louis Courajod (1841-1896) bouleversa l'image traditionnelle de la Renaissance et donna une forte impulsion aux études sur la production nationale, dont il s'agissait de mettre en lumière la «francité». L'idée que l'influence d'un art étranger avait pu provoquer une rupture dans la tradition française suscita une forte résistance de la part d'une histoire de l'art qui revendiquait ostensiblement pour la France un rôle de premier plan dans les mouvements qui conduisirent à l'élaboration de l'art moderne. Ainsi, de nombreuses études s'attachèrent à une révision radicale de l'image traditionnelle de 
l'évolution des styles, et tentèrent de doter l'art français d'un nouveau statut parmi les productions des autres nations européennes en niant toute "contamination " avec d'autres traditions figuratives.

Dans une France qui était en train de redécouvrir une Renaissance toute nationale, d'essence nordique et antilatine, l'œuvre de Müntz rencontrait nécessairement de fortes résistances. Alors qu'il était une figure centrale à un niveau international, Müntz occupait une position de plus en plus marginale dans le champ de l'historiographie française de l'art, qui précipitera plus tard la rapide obsolescence de son œuvre. S'il est vrai que Müntz jouit d'une importante reconnaissance officielle, et qu'il exerça une influence décisive dans l'évolution de la discipline en tant que directeur de nombreuses initiatives éditoriales, il n'atteindra jamais une position prééminente dans l'enseignement universitaire. Les vicissitudes de son professorat à l'École des BeauxArts sont tout à fait révélatrices d'un isolement de plus en plus marqué. Quand en 1893 Taine mourut et que s'ouvrit le débat sur sa succession, la direction de l'École des Beaux-Arts choisit non pas Müntz, qui l'avait suppléé pendant plusieurs années, mais le critique et journaliste Louis de Fourcaud, connu pour les implications nationalistes de ses théories, et qui professa un enseignement fort proche des doctrines de Courajod sur les origines de la Renaissance ${ }^{28}$.

Müntz mourut en 1902, l'année même où l'exposition des Primitifs français (1904) commençait à s'organiser sous la direction d'Henri Bouchot ${ }^{29}$. C'était de l'héritage de Courajod que Bouchot et ses collaborateurs se réclamaient : de cette idée de la primauté des pays du Nord que Müntz avait combattu tout au long de sa carrière.

En 1904, alors que l'exposition était encore en cours, Warburg fit paraitre en italien dans la Rivista d'arte un article intitulé "Per un quadro fiorentino che manca all'esposizione dei Primitivi francesi $»^{30}$, qui attirait l'attention des chercheurs sur l'Adoration des Mages de Benedetto Ghirlandaio, découverte par Paul Mantz à Aigueperse, en Auvergne. Warburg y insistait de façon polémique sur les circulations multiples d'artistes et de modèles entre France, Flandres et Italie, et relevait le «style étrangement ultramontain » du tableau qui, en l'absence de l'inscription déchiffrée par Mantz, aurait rendu difficile d'établir si son auteur était «un flamand ou un français toscanisant ou bien un italien peignant "à la française" ». En stigmatisant le nationalisme des organisateurs de l'exposition, Warburg se situait clairement dans le champ qui avait été celui de Müntz.

30 Cette proximité intellectuelle de Warburg et Müntz doit être prise en compte au moment où il s'agit d'aborder la question de la réception extrêmement problématique de l'œuvre de Warburg en France. Dans ce cadre d'une réception difficile, tardive, de la méthode de Warburg en dans l'historiographie de l'art de langue française, il y a une exception intéressante : l'historien de l'art Jacques Mesnil, d'origine belge, qui, ami de Warburg, intègre précocement des références warburgiennes dans ses écrits. Mesnil est d'ailleurs un cas singulier : un chercheur qui publie en français mais qui est une figure d'apatride de la recherche, tant qu'il est difficile de le situer effectivement dans une tradition française d'études ${ }^{31}$.

31 Mesnil, lecteur attentif de l'essai de Warburg sur la Naissance de Venus et le Printemps qui devait marquer durablement sa propre analyse de l'œuvre de Botticelli ${ }^{32}$ partageait avec celui-ci un intérêt marqué pour le problème des relations artistiques entre le Nord et le Sud de l'Europe à la Renaissance. Il l'aborda dans différents articles, dans l'ouvrage L'Art au Nord et au Sud des Alpes à l'époque de la Renaissance, paru en 1911, 
ainsi que dans son intervention au Xe Congrès international d'histoire de l'art (1912) le même où Warburg présenta ses recherches sur les fresques du Palazzo Schifanoia ${ }^{33}$.

Mesnil prit part aux travaux de la Bibliothèque Warburg, dont il relata les résultats dans des articles parus dans la Gazette des Beaux-Arts (1926-29) ${ }^{34}$. Il aura donc servi d'intermédiaire pour une réception qui en France aura lieu beaucoup plus tard. Une précision s'impose d'ailleurs : ce que Mesnil retient de l'œuvre de Warburg n'est pas la réflexion sur l'iconologie mais plutôt l'attention aux conditions sociales de la production des œuvres : la partie de l'œuvre de Warburg qui ouvre vers une histoire sociale de l'art.

En posant la question de la réception de Warburg en France il faut surtout éviter l'écueil d'une lecture téléologique, qui nous amènerait à être surpris qu'un auteur qui nous intéresse, dans la mesure où il semble répondre à une série de questions ouvertes de notre histoire de l'art, ait eu un impact tardif et inégal sur une tradition d'études qui, comme l'historiographie de l'art française de la première partie du $\mathrm{XX}^{\mathrm{e}}$ siècle, se posait des questions complètement différentes.

Warburg a eu comme principal interlocuteur en France un savant, Eugène Müntz qui, par sa lecture de la Renaissance en France et en Europe, était plutôt isolé dans le cadre de l'histoire de l'art française telle qu'elle était en train de s'élaborer au tournant du $\mathrm{XX}^{\mathrm{e}}$ siècle - un savant dont certains de ses contemporains estimaient qu'il était plus proche de la tradition historiographique allemande que de la française.

Ainsi, il est peut-être utile de poser ce problème de la réception de l'œuvre de Warburg - et aussi de la méthode que Warburg a inaugurée et d'autres ont développée - en France dans les termes d'un questionnement sur les échanges entre traditions nationales en sciences humaines ${ }^{35}$, sur leur relative étanchéité et sur les représentations qui leur sont attachées. L'histoire de l'art française du $\mathrm{XX}^{\mathrm{e}}$ siècle peut être considérée comme une tradition singulière, spécifique? Quels en seraient alors les éléments constitutifs? Quelles résistances y rencontra l'œuvre de Warburg? Mais aussi, en d'autres termes, qu'est-ce qui fit de Müntz, à la fin de sa carrière, un savant isolé ?

Répondre à ces questions demanderait de procéder à une prise en compte critique du discours sur l'art en France et en Europe sur au moins deux siècles. L'analyse des relations intellectuelles que Warburg entretint avec cette figure singulière de l'historiographie française, Eugène Müntz, apporte peut-être quelques premiers éléments de réponse.

\section{NOTES}

1. Cette lettre a été publiée en partie dans Ernst Gombrich, Aby Warburg, an Intellectual Biography, Oxford, 1986, p. 143 et intégralement, en italien, par Maurizio Ghelardi : Aby Warburg, «La storiografia artistica : una lettera a Adolph Golschmidt », dans id., La rinascita del paganesimo antico e altri scritti, (1889-1914), éd. par M. Ghelardi, Turin, 2004, p. 359-364, ici p. 363. 
2. Sur la carrière et l'œuvre de Müntz, voir: Jules Maurice, "Notice nécrologique sur Eugène Müntz (1845-1902) », Bulletin de la Société Nationale des Antiquaires de France, 1906, p. 67-84 ; Camille Enlart et Georges de Manteyer, E. Müntz. Notice biographique par C. Enlart. Bibliographie par G. de Manteyer, Rome, 1903; Henri Cordier, «Eugène Müntz. In memoriam », Revue des traditions populaires, t. XVIII, 1903, p. 620-625 ; André Girodie, « Eugène Müntz », Revue alsacienne illustrée, t. IV, 1902, p. 65-74 ; Louis Dimier, «Eugène Müntz », Gazette des Beaux-Arts, t. XXIX, 1903, p. 42-47. Plus récemment: Michel Espagne, L'Histoire de l'art comme transfert culturel. L'itinéraire d'Anton Springer, Paris, 2009, p. 238-242; Michela Passini et François-René Martin, «Ressentiment politique, affinités intellectuelles. Eugène Müntz et l'histoire de l'art allemande », Annali della Scuola Normale Superiore di Pisa, t. I, 2009, p. 227-254.

3. Bibliothèque nationale de France, Eugène Müntz, correspondance reçue, NAF 11314, 10 lettres.

4. Vom Arsenal zum Laboratorium (1927) a été publié pour la première fois par Maurizio Ghelardi, en traduction italienne, dans Aby Warburg, La rinascita del paganesimo antico e altri scritti, op. cit., p. 6-16.

5. Aby Warburg, Wappen, Stammbäume und Inventare als Hilfsmittel der Kunstgeschichte, dans Offizielles Berich ueber die Verhandlungen des VII. Internationalen Kunsthistorischen Kongresses in Innsbruck (9.-12. September 1902), Berlin, s.d, p. 92-97.

6. Voir notamment Eugène Müntz, Les Arts à la cour des papes pendant le XV et le XVI siècle. Recueil de documents inédits tirés des archives et des bibliothèques romaines, 3 vol., Paris, 1878-1882.

7. Voir la correspondance qu'il entretint avec Müntz: Bibliothèque nationale de France, Correspondance d'Eugène Müntz, NAF 11313.

8. Eugène Müntz, brouillon de lettre, s.d., destinataire inconnu, Bibliothèque Nationale de France, Correspondance d'Eugène Müntz, NAF 11315, f. 272.

9. Maurizio Ghelardi, «Introduzione », dans Jacob Burckhardt, L'arte italiana del Rinascimento. I collezionisti, Venise, 1995, p. XIII.

10. Je me permets de renvoyer à mon ouvrage : Michela Passini, La fabrique de l'art national. Le nationalisme et les origines de l'histoire de l'art en France et en Allemagne (1870-1933), éditions de la Maison des sciences de l'homme - Centre allemand d'histoire de l'art, 2012.

11. Note manuscrite, datée 3 mars 1866, Bibliothèque Nationale de France, Eugène Müntz, Documents sur l'histoire des arts, NAF 21500, ff. 775-776.

12. Henri Cordier, « Eugène Müntz. In memoriam », op. cit., p. 621.

13. Peter Schmidt, Aby Warburg und die Ikonologie. Mit einem Anhang von Dieter Wuttke, Wiesbaden, 1993.

14. Bibliothèque Nationale de France, Correspondance d'Eugène Müntz, NAF 11310.

15. Eugène Müntz, Études sur l'histoire de la peinture et de l'iconographie chrétienne, Paris, G. Fischbacher, 1882.

16. Id., Histoire de l'art pendant la Renaissance, vol. I : la tapisserie est reproduite, en couleurs, dans le frontispice.

17. Ces articles furent réunis dans le recueil Eugène Müntz, Les légendes du moyen âge dans l'art de la Renaissance, Vannes, Impr. de Lafolye, 1892.

18. Henry Thode, Franz von Assisi und die Anfänge der Kunst der Renaissance in Italien, Berlin, 1885 ; trad. fr. par Gaston Lefèvre (pseud. de Théodore de Wyzewa), Saint François d'Assise et les origines de l'art de la Renaissance en Italie, 2 vol., Paris, 1909. Pour sa correspondance avec Müntz, voir : Bibliothèque Nationale de France, Correspondance d'Eugène Müntz, NAF 11312.

19. Josef Strzygowski, Iconographie der Taufe Christi. Ein Beitrag zur Entwicklungsgeschichte der christlichen Kunst, Munich, 1885. Pour sa correspondance avec Müntz, voir: Bibliothèque Nationale de France, Correspondance d'Eugène Müntz, NAF 11311.

20. Émile Mâle, L'Art religieux du XIII 'siècle en France, étude sur l'iconographie du Moyen-Âge et sur ses sources d'inspiration, Paris, 1898. 
21. Voir note 14.

22. Sur Morelli, voir Michela Passini, "Giovanni Morelli", dans Michel Espagne et Bénédicte Savoy (dir.), Dictionnaire des historiens de l'art allemands, Paris, Éditions du CNRS, 2010, p. 157-164, avec bibliographie.

23. Eugène Müntz, «La Nécessité des études iconographiques », dans Peter Schmidt, Aby Warburg und die Ikonologie. Mit einem Anhang von Dieter Wuttke, op. cit., p. 56-57.

24. Ibid., p. 56.

25. Voir n. 14 ; lettres du 31 décembre 1883 et du 20 juin 1886.

26. Eugène Müntz, « Programme », dans Peter Schmidt, Aby Warburg und die Ikonologie. Mit einem Anhang von Dieter Wuttke, op. cit., p. 83-86.

27. Roland Recht, «L'iconologie d'Aby Warburg: enquête sur son origine ", communication au colloque Survivance d'Aby Warburg. Sens et destin d'une iconologie critique, ENSBA (Paris) et CAPC (Bordeaux), 19-20 mai 2011, sous la direction de Sabine Forero-Mendoza et Bertrand Prévost.

28. Samuel Rocheblave, Louis de Fourcaud et le mouvement artistique en France de 1875 à 1914, Paris, 1926, p. 216-220.

29. Sur cette exposition, voir: Dominique Thiébaut, François-René Martin, Philippe Lorentz (dir.), Primitifs français, découvertes et redécouvertes, cat. expo., Paris, Musée du Louvre, 2004.

30. Aby Warburg, «Per un quadro fiorentino che manca all'esposizione dei Primitivi francesi », Rivista d'Arte, t. II, n 5, 1904, p. 84-86.

31. Je me permets de renvoyer à mon article: Michela Passini, "Jacques Mesnil», dans Ph. Bordes (dir.), Histoire sociale de l'art: une anthologie critique, à paraître aux éditions de l'INHA et des Presses du Réel.

32. Jacques Mesnil, Botticelli, Paris, A. Michel, 1938.

33. Id., L'Art au Nord et au Sud des Alpes à l'époque de la Renaissance. Études comparatives, ParisBruxelles, G. Van Oest, 1911.

34. Id., "La bibliothèque Warburg et ses publications ", Gazette des Beaux-Arts, 14, 1926, p. 237. Voir aussi : Id., « Conférences de la Bibliothèque Warburg », ibid., 1, 1929, p. 319-320.

35. Johan Heilbron, «Repenser la question des traditions nationales en sciences sociales ", dans Gisèle Sapiro, L'espace intellectuel en Europe. De la formation des États-nations à la mondialisation, XIX ${ }^{e}$ XXI siècle, Paris, La Découverte, 2009, p. 309-318.

\section{INDEX}

Mots-clés : Historiographie, tradition, réception, panni, motifs, échanges artistiques, France, Allemagne

\section{AUTEUR}

\section{MICHELA PASSINI}

Chercheuse au CNRS (Institut d'histoire moderne et contemporaine). Spécialiste de l'historiographie de l'art et de l'histoire des musées et du patrimoine, elle prépare une histoire transnationale des expositions dans les musées européens et nord-américains (1900-1940). Ses publications les plus récentes sont La fabrique de l'art national. Le nationalisme et les origines de 
l'histoire de l'art en France et en Allemagne (1870-1933), Paris, 2012, Sarah Linford et Michela Passini (dir.), Le Génie dans l'art. Anthologie des écrits esthétiques et critiques de Gabriel Séailles, Paris, 2011; Maurice Barrès, La grande pitié des églises de France, (édition commentée avec Michel Leymarie), Paris, 2012. 